OPEN ACCESS

Edited by: Giuseppe Calamita, University of Bari Aldo Moro, Italy

Reviewed by: Maria Grano,

University of Bari Aldo Moro, Italy

Claudia Penna,

University of Turin, Italy

*Correspondence:

Jiake Xu

jiake.xu@uwa.edu.au

Jun Zou

junzou@sus.edu.cn

Specialty section:

This article was submitted to

Cellular Biochemistry,

a section of the journal

Frontiers in Cell and Developmental

Biology

Received: 17 February 2021

Accepted: 06 April 2021

Published: 04 May 2021

Citation:

Liu L, Guo J, Chen X, Tong X, Xu J and Zou J (2021) The Role of Irisin

in Exercise-Mediated Bone Health.

Front. Cell Dev. Biol. 9:668759.

doi: 10.3389/fcell.2021.668759

\section{The Role of Irisin in Exercise-Mediated Bone Health}

\author{
Lifei Liu',2, Jianmin Guo', Xi Chen ${ }^{3}$, Xiaoyang Tong ${ }^{1}$, Jiake $X u^{4 *}$ and Jun Zou ${ }^{1 *}$ \\ ${ }^{1}$ School of Kinesiology, Shanghai University of Sport, Shanghai, China, ${ }^{2}$ Department of Rehabilitation, The People's Hospital \\ of Liaoning Province, Shenyang, China, ${ }^{3}$ School of Sports Science, Wenzhou Medical University, Wenzhou, China, ${ }^{4}$ School \\ of Biomedical Sciences, University of Western Australia, Perth, WA, Australia
}

Exercise training promotes physical and bone health, and is the first choice of non-drug strategies that help to improve the prognosis and complications of many chronic diseases. Irisin is a newly discovered peptide hormone that modulates energy metabolism and skeletal muscle mass. Here, we discuss the role of irisin in bone metabolism via exercise-induced mechanical forces regulation. In addition, the role of irisin in pathological bone loss and other chronic diseases is also reviewed. Notably, irisin appears to be a key determinant of bone mineral status and thus may serve as a novel biomarker for bone metabolism. Interestingly, the secretion of irisin appears to be mediated by different forms of exercise and pathological conditions such as diabetes, obesity, and inflammation. Understanding the mechanism by which irisin is regulated and how it regulates skeletal metabolism via osteoclast and osteoblast activities will be an important step toward applying new knowledge of irisin to the treatment and prevention of bone diseases such as osteolysis and other chronic disorders.

Keywords: irisin, exercise, bone health, skeleton, mechanical force

\section{INTRODUCTION}

Exercise training is well known to have beneficial effects on physical fitness and bone health. Physical activity reduces the risk of many chronic diseases and aging-related disorders, such as diabetes mellitus, hypertension, obesity, and osteoporosis (Farmer, 2019). Lack of physical activity, such as resembling bedridden patients, causes a lack of mechanical stimulation, leading to an imbalance of bone formation and resorption and a speedy loss of bone mass (Benedetti et al., 2018). Proper high impact physical activity enables bone to respond positively, and improve the renew of bone metabolism, bone mineral density (BMD) and structural properties in the loaded bone regions and whole body (Beck et al., 2017; Gomez-Bruton et al., 2017; Okubo et al., 2017). Meanwhile, regular physical activity ameliorates bone health status and reduces the risk of trauma fragility fracture and secondary functional disfunction (Kemmler et al., 2015; Troy et al., 2018).

Irisin is a newly discovered peptide hormone of 112 amino acids, which is the extracellular domain of a transmembrane protein fibronectin type III domain-containing 5 (FNDC5). FNDC5 and its cleaved circulating form irisin are positively correlated with an active lifestyle (Tenorio et al., 2017). Vigorous-intensity physical activity had a high serum level of irisin (Morelli et al., 2020), which positively related to bone mechanical properties (Zhang et al., 2020). Furthermore, treatment with irisin is found to improve BMD and biomechanical properties in murine models (Colaianni et al., 2015). Here we review irisin as a key factor linking exercise and bone health and discuss the different roles of irisin in musculoskeletal system and some chronic disease conditions mediating bone metabolism in the context of exercise. 


\section{ROLE OF EXERCISE IN REGULATING BONE METABOLISM}

Exercise regulates bone metabolism, mainly through direct action (mechanical force) and indirect action (nerve and hormone regulation). Mechanical force acts on bone via ground reaction and muscle contraction forces (Usui et al., 2003). Loss of mechanical force, such as hindlimb murine, the trabecular bone volume was reported significantly reduced, and subsequent reloading results in a significant increase in trabecular bone volume (Colaianni et al., 2017; Cunningham et al., 2018). The central nervous system, especially the cerebral cortex and cerebellum regulates the neuromuscular and musculoskeletal systems, leading to the activation and precise adjust of bones (Cardozo and Graham, 2017). While, the hypothalamus influences bone is mainly by regulating the secretion of pituitary hormones (Dimitri and Rosen, 2017). Additionally, the autonomic nervous system, including the sympathetic (negative regulator) and parasympathetic (positive regulator) nervous systems has also been found to affect bone metabolism (Houweling et al., 2015; Idelevich and Baron, 2018).

Irisin is found to regulate bone cell metabolism by mechanical force (Storlino et al., 2019), and to increases both cortical and trabecular BMD subsequently (Colaianni et al., 2017). Additionally, muscle strength is found to be enhanced after irisin treatment (Reza et al., 2017b). Moreover, irisin participates in the regulation process of the hypothalamus and the autonomic nervous system (Scalzo et al., 2014; Poretsky et al., 2017) and interacts with hormones related to bone metabolism (Kim and Kim, 2018).

\section{Regulation of Bone Cell Metabolism}

Bone physiological functions are mainly maintained by the activity of bone cells such as osteoblasts, osteoclasts and osteocytes (Clarke, 2008). Osteoblast-led bone formation and osteoclast-led bone resorption maintain bone homeostasis simultaneously (Kular et al., 2012). Osteocytes are the chief mechanosensory cells, and stellate cells embedded in calcified bone matrix, which accounts for over $90 \%$ of the bone cells (Klein-Nulend et al., 1995, 2012; Bonewald, 2011).

During high impact and rapid loading physical exercise (Beck et al., 2017), ground reaction forces exert mechanical forces on the bones (Usui et al., 2003), and the deformation of the bone matrix drives an interstitial fluid flow, which surrounds the osteocytes (Knothe Tate et al., 2000). Stimulated by the fluid mechanical signals, osteocytes regulate a cascade of biochemical responses (Kulkarni et al., 2012). Simultaneously, osteocytes convert mechanical strain into regulatory signals to stimulate the adaptive response of osteoclasts, thereby activating the bone resorption and subsequent formation process (Bonewald, 2011; Troy et al., 2018).

Irisin prevents the decrease of living osteocytes and the increase of empty cavities due to disuse (Storlino et al., 2019). Previous studies have shown that mechanical load leads to an increase of Wnt protein in osteocytes, thereby activating the classic Wnt signaling pathway, adjusting its sensitivity to mechanical load in the feedback loop (Tu et al., 2012).
Corresponding, FNDC5 knockdown cells show downregulated Wnt expression (Ma et al., 2019). One study reveals that irisin increases survival of osteocytes by activating the mitogenactivated protein kinase (MAPK) extracellular signal-regulated kinase 1 (ERK1) and ERK2, which increases the expression of the transcription factor activating transcription factor 4 (ATF4) through an ERK-dependent pathway. Besides, irisin has been found to inhibit apoptosis in osteocytes (Storlino et al., 2019).

The osteoblast is the target of irisin as well. Irisin enhances osteoblast differentiation, proliferation, mineralization, and upregulates the expression of transcription regulators, such as runt-related transcription factor-2 (Runx2) and osterix (Colaianni et al., 2015; Zhang et al., 2017). Recent studies reveal that the osteogenic effect is mediated by irisin through activation of the p38 MAPK and ERK (Qiao et al., 2016), and differentiation promotion effect might be associated with activation of AMPK AMP-activated protein kinase (AMPK)- $\alpha$ signaling (Ye et al., 2020) and $W n t / \beta$-catenin pathway (Robinson et al., 2006). Other studies indicate that the proliferation of osteoblasts can be promoted by irisin via enhancing aerobic glycolysis (Zhang et al., 2018), and the osteoblast apoptosis is suppressed by irisin via upregulating nuclear factor E2-related factor 2 (Nrf2), inhibiting pyrin domain containing protein 3 (NLRP3) inflammasome and lowering the content of inflammatory factors, which cause the reduction of the incidence of postmenopausal osteoporosis (Xu et al., 2020). Besides, recent studies show that irisin not only stimulates autophagy but also downregulates a senescence effector p21 to promote osteoblastogenesis and maintain the activity of osteoblast (Chen et al., 2020; Colaianni et al., 2021).

Osteoclast formation and differentiation are significantly reduced by irisin treatment (Zhang et al., 2017), and nuclear factor $\kappa \mathrm{B}(\mathrm{NF}-\kappa \mathrm{B})$ ligand (RANKL) was found to be a key factor (Kawao et al., 2018). Irisin suppresses the receptor activator of RANKL/nuclear factor of activated T cells (NFAT) c1 pathway, thereby inhibits osteoclast formation in mouse bone marrow cells (Zhang et al., 2017; Kawao et al., 2018). A study of FNDC5 knockout mice indicates that the higher expression of RANKL and increased number of osteoclasts cause a decrease in bone strength and bone mass (Luo et al., 2020).

\section{Potential Role in Regulating the Crosstalk Between Muscle and Bone}

Irisin was originally found to be secreted by muscle cells (Bostrom et al., 2012), and muscle contraction increases irisin secreted during exercise (Archundia-Herrera et al., 2017). Muscle is the major tissue of irisin expression, although it is also expressed in small amounts in bone, brain and other tissues (Colaianni et al., 2015). Muscle force itself is capable of providing sufficient stimulation to make bones respond (Judex and Rubin, 2010). Some investigations show that bone density is significantly related to muscle strength (Nordstrom et al., 1998). For example, the jumping force is positively correlated with increased bone mineral content (BMC) in the tibial cortex (Zengin et al., 2017), while, the decline of muscle function, such as sarcopenia, can lead to bone mass loss (Bonewald, 2019). Besides, the optimization of muscle strength, balance, and mobility brought by exercise can minimize the risk of falls and subsequent 
fractures, which is especially important for people at high risk of falls (Beck et al., 2017).

Irisin is positively correlated with muscle mass (Kim et al., 2016) and muscle strength, such as hand grip strength and leg strength (Martinez Munoz et al., 2019). Studies have established that the injection of irisin in murine induces significant hypertrophy of skeletal muscle and enhances muscle strength (Reza et al., 2017b), even reduce necrosis and fibrotic tissue (Reza et al., 2017a). The effect of irisin on hypertrophy is due to muscle stem cell activation and enhanced protein synthesis (Reza et al., 2017b). Muscle movement also induces peroxisome proliferatoractivated receptor- $\gamma$ coactivator $1 \alpha(\mathrm{PGC} 1 \alpha)$, a transcriptional coactivator (Handschin and Spiegelman, 2008). PGC1 $\alpha$ in muscle is reported to stimulate an increase in FNDC5 expression in vitro and in vivo (Bostrom et al., 2012). A recent study indicates that exercise increases mitochondrial fission and selective autophagy by PGC1a/FNDC5/irisin pathway, and promotes recovery of ischemic muscle (He et al., 2020). These studies suggest that irisin may play an important role in exercise relieving skeletal muscle atrophy, thereby maintaining bone load and bone mass.

\section{Exercise-Mediated Cell Factors in Bone Metabolism}

Some cell factors are regulated by irisin during exercise to balance bone metabolism. Bone cells, especially osteocytes modify the production of a large number of signaling molecules when triggered by mechanical stimulation (Janik et al., 2018). At the same time, some central nervous system related hormones are also changed due to exercise, affecting bone metabolism (Kim and Kim, 2018; Liu et al., 2021).

Sclerostin is a protein produced by osteocytes, and causes endogenous inhibition of bone formation, which regulates bone remodeling (van Bezooijen et al., 2004). People who have more physical activities are tested as less sclerostin, and regular physical training results in a significant decrease of sclerostin level (Cheung and Giangregorio, 2012; Janik et al., 2018). Sclerostin is found to be a Wnt antagonist and blocks the Wnt/ $\beta$-catenin signaling pathway (Singh et al., 2019). Therefore, sclerostin inhibitors can be expected to increase osteoblastogenesis (Lewiecki, 2011). Researches indicate that circulating irisin and sclerostin are highly negatively correlated (Colaianni et al., 2017). Additionally, irisin treatment inhibits the increase of sclerostin and restores osteoblastogenesis (Colaianni et al., 2017). On the contrary, one study reveals that irisin treatment upregulates sclerostin expression in osteoin-like cells (MLO-Y4) in a dose-dependent manner, and knockout of FNDC5 prevents ovariectomy mice from BMD loss by inhibiting osteolysis and bone resorption. The conflicting conclusion may be related to the different regulatory effects of irisin on osteogenesis and osteoclastogenesis under different conditions (Kim et al., 2018). This situation may be similar to the bidirectional regulation of bone by parathyroid hormone, in which intermittent administration leads to bone formation, while continuous administration causes bone loss (Rattanakul et al., 2003).

Leptin has been reported to regulate bone metabolism mainly by acting on the brain, especially via the hypothalamus and sympathetic nervous system (Motyl and Rosen, 2012; Reid et al., 2018). Leptin is a negative regulator of bone, and multiple lines of evidence show that high bone mass phenotype can be caused by leptin gene deletion accompanied with a massive increase in bone formation (Ducy et al., 2000; Karsenty, 2006). In addition, intraventricular injection of leptin reduces bone mass and volume by increasing osteoclast activity (Ducy et al., 2000). Exercise decreases leptin level, evidence suggests that running wheels exercise reduces circulating leptin levels in both adults and adolescents rats (Soch et al., 2016). Notably, circulating irisin and leptin are positively related in children and adults (PalaciosGonzalez et al., 2015; Pena-Bello et al., 2016). In a study of rats, intraperitoneal irisin injections decreased the leptin level in circulation (Tekin et al., 2017). Interestingly, leptin has also been found to up-regulate the expression of FNDC5 through a nitric oxide-dependent mechanism (Rodriguez et al., 2015).

Brain-derived neurotrophic factor (BDNF) is found to be more expressed in bone than in the brain, as a neuroprotective factor (Camerino et al., 2016; Kowianski et al., 2018), and is involved in regulating the formation and fracture healing process of bone (Kilian et al., 2014). A report shows that BDNF promotes bone marrow mesenchymal stem cells osteogenesis by binding to the tropomyosin-related kinase $\mathrm{B}(\mathrm{TrkB})$ receptor, downstream Erk1/2 phosphorylation, and BDNF indirectly promotes osteogenesis by increasing neurogenesis as well (Liu et al., 2018). Exercises may have a regulatory effect on BDNF secretion, as studies reveal that a 3-months crossfit training increases BDNF level in young people (Murawska-Cialowicz et al., 2015), while sedentary rats have lower BDNF than the exercised ones, both young and aged (Belviranli and Okudan, 2018). Analogously, overexpression of irisin significantly upregulates BDNF expression, while irisin interference significantly downregulated the level of BDNF (Huang et al., 2019). To date, there is a lot of uncertainty in the role of irisin in cell factors expression level, which requires further research.

\section{ROLE OF EXERCISE IN REGULATING CHRONIC DISEASES}

Some chronic diseases, such as diabetes mellitus (Mahapatra et al., 2016), inflammatory bowel diseases (Ali et al., 2009), hyperthyroidism (Novack, 2003), and relative adiposity (Dolan et al., 2017) are shown to be associated with low BMD and bone loss (Amin et al., 2011; Dimitri and Rosen, 2017). Exercise is the first line for treating various diseases, which also improves the prognosis and complications (Jin et al., 1999). The previous studies have established that exercise and irisin promote osteogenesis in some chronic diseases (Dieli-Conwright et al., 2018; Palermo et al., 2019).

\section{Diabetes Mellitus}

Type 1 diabetes mellitus (T1DM) has an important link with osteoporosis which begins in childhood, and leads to lower peak bone mass and high risk of osteoporotic fractures in adults (Weber et al., 2015; Devaraja et al., 2020). Poor glycemic control and glycated hemoglobin $(\mathrm{HbA1c})$ are found to be negatively 
TABLE 1 | Irisin regulates bone metabolism by different factors.

\begin{tabular}{|c|c|c|}
\hline Factor & Action & References \\
\hline MAPK & $\begin{array}{l}\text { Improve osteocyte } \\
\text { survival, inhibit } \\
\text { apoptosis }\end{array}$ & Storlino et al., 2019 \\
\hline P38 MAPK and ERK & $\begin{array}{l}\text { Improve osteoblast } \\
\text { osteogenic }\end{array}$ & Qiao et al., 2016 \\
\hline Nrf2 & $\begin{array}{l}\text { Inhibit osteoblast } \\
\text { apoptosis }\end{array}$ & Xu et al., 2020 \\
\hline $\mathrm{AMPK}-\alpha$ & $\begin{array}{l}\text { Improve osteoblast } \\
\text { differentiation }\end{array}$ & Ye et al., 2020 \\
\hline RANKL & $\begin{array}{l}\text { Inhibit osteoclast } \\
\text { differentiation }\end{array}$ & $\begin{array}{l}\text { Zhang et al., 2017; } \\
\text { Kawao et al., } 2018\end{array}$ \\
\hline Sclerostin & $\begin{array}{l}\text { Improve osteocyte } \\
\text { survival and } \\
\text { osteoblastogenesis }\end{array}$ & $\begin{array}{l}\text { Colaianni et al., } \\
2017 \text {; Kim et al., } \\
2018\end{array}$ \\
\hline Muscle stem cell-derived factors & $\begin{array}{l}\text { Increase muscle mass } \\
\text { and strength }\end{array}$ & Reza et al., 2017b \\
\hline PI3K & $\begin{array}{l}\text { Improve insulin } \\
\text { sensitivity, reduce } \\
\text { fasting glycemia }\end{array}$ & Liu et al., 2015 \\
\hline$N F-\kappa B$ & Inhibit inflammation & $\begin{array}{l}\text { Mazur-Bialy et al., } \\
2017\end{array}$ \\
\hline
\end{tabular}

correlated with BMD (Fuusager et al., 2019). Studies of diabetic mellitus rats demonstrate that running exercise increases the irisin level, glycemic control, bone mass and muscle strength, probable due to the activation of the $\mathrm{Wnt} / \beta$-catenin signaling pathway and decreased systemic inflammatory process (Andrade et al., 2018; Yang et al., 2018; Sadeghipour et al., 2020).

Irisin levels are indicated significantly correlated negatively with $\mathrm{HbA1c}$, years of diabetes, and positively associated with better glycemic control and bone health in TD1M children (Kurdiova et al., 2014; Faienza et al., 2018; Gouda et al., 2018). Furthermore, persistent subcutaneous perfusion of irisin improves insulin sensitivity, reduces fasting glycemia by inhibiting gluconeogenesis via phosphoinositide 3-kinase
$(\mathrm{PI} 3 \mathrm{~K}) /$ serine/threonine kinase $(\mathrm{Akt}) /$ forkhead box transcription factor O1 (FOXO1) mediated phosphoenolpyruvate carboxykinase (PEPCK) (Liu et al., 2015). A recent study shows that irisin regulates glucose metabolism by promoting hepatic glycolysis and inhibiting hepatic gluconeogenesis (Yang et al., 2020). Therefore, irisin may improve bone metabolism of diabetic patients by regulating glycemic levels through exercise.

\section{Obesity}

Some studies indicate that overweight children have lower bone mass than normal weight children, relative to their size and poorer bone structure parameters (Goulding et al., 2000; Farr et al., 2010). Another report shows bone strength is related to lean mass rather than fat mass (Bogl et al., 2011), and excess fat seems to limit the effect of lean mass on bone maturation (Farr and Dimitri, 2017).

Exercise can alleviate bone loss caused by obesity. A recent study suggests that subsequent 8 weeks of swimming relieves the reduced $\mathrm{BMD}$, bone microstructure, and bone metabolic factors on obese rats (Kang et al., 2019). In obese breast cancer survivors, whole body and trochanter BMD have an upward trend after a 4-months exercise (Dieli-Conwright et al., 2018). Although the relevance to gender is unclear (Anastasilakis et al., 2014; Ruan et al., 2019), circulating irisin is shown positive correlated with adiposity indices, such as percent body fat and fat mass (Jang et al., 2017). Notably, a 6-months moderate physical exercise increases the irisin level, decreases body mass index and waist circumferences in obese men (Rashid et al., 2020). Therefore, irisin may be an important factor in maintaining the bone health of obese people.

\section{Inflammation}

Lack of exercise also activates the inflammatory pathway network, which promotes the development of a cluster of diseases (Pedersen, 2009). Chronic inflammatory diseases cause

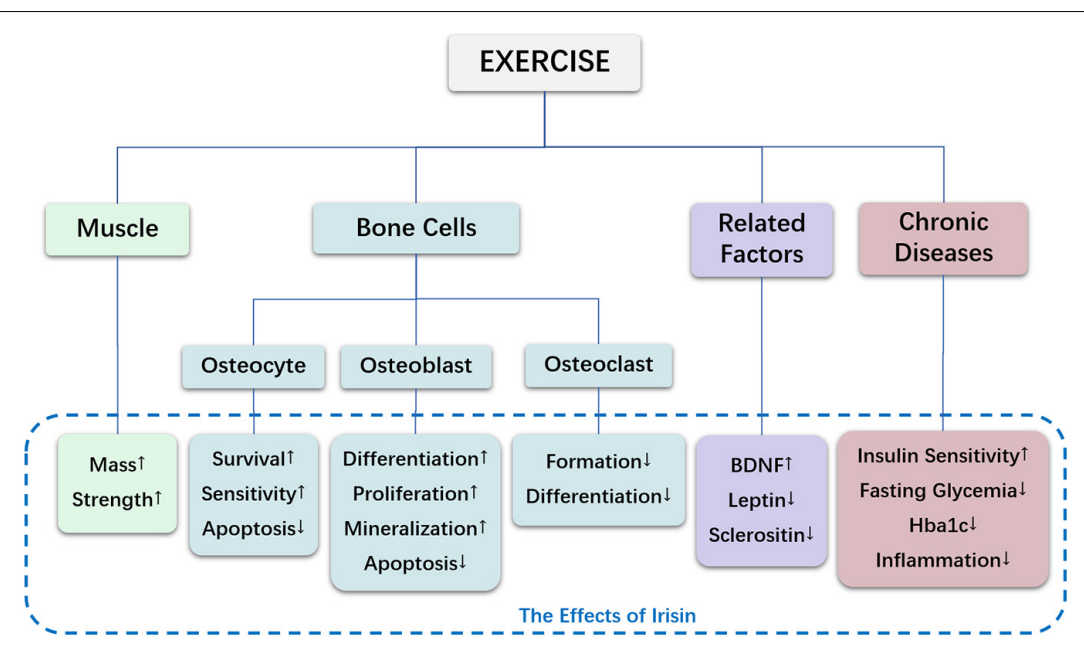

FIGURE 1 | The effects of irisin in exercise-mediated bone metabolism. 
excessive bone absorption and impaired bone formation, leading to periarticular and systemic bone loss (Straub et al., 2015; Metzger et al., 2019). Exercise promotes irisin expression and induces anti-inflammatory effects (Wiecek et al., 2018). Serum irisin levels are negatively correlated with inflammation-related symptoms, such as disease duration, severity evaluation, and stiffness duration in rheumatoid arthritis patients (Gamal et al., 2019). Anti-inflammatory property of irisin is associated with the downregulation of the Toll-like receptor 4 (TLR4)/myeloid differentiation primary response protein 88 (MyD88) downstream pathway and decreased the phosphorylation of NF- $\kappa \mathrm{B}$, consequently decreased phosphorylation and activation of crucial pro-inflammatory cytokines (Mazur-Bialy et al., 2017). Recently, in the study of a rat model of disuse osteoporosis, irisin treatment increases the bone formation rate of unloading hindlimbs and reduce the expression of pro-inflammatory factors such as tumor necrosis factor (TNF)- $\alpha$ and Interleukin (IL)-17 (Metzger et al., 2020).

\section{POTENTIAL DEVELOPMENT OF IRISIN AS THERAPEUTIC AGENT AND BIOMARKERS FOR BONE DISEASES}

Increasing the level of physical activity is considered to be the preferred non-pharmacological intervention for the prevention and treatment of chronic bone diseases (Compston et al., 2017). Some resistance trainings during early life improve BMD and bone structural properties, and have a direct preventive effect on bone diseases in later life (Gomez-Bruton et al., 2017), and irisin also plays a key role in this process (Elizondo-Montemayor et al., 2018). Although different types of exercise training have conflicting results on irisin, most studies suggest that highmoderate intensity (Tsuchiya et al., 2014; Rashti et al., 2019; Torre-Saldana et al., 2019) and resistance exercises (Nygaard et al., 2015; Tsuchiya et al., 2015; Kim et al., 2016) lead to more significantly enhanced in irisin level, and improve bone loss in patients with osteoporosis (Watson et al., 2019). This means that irisin has become a new target in promoting bone health, and the possibility of treating some bone diseases.

Irisin is also considered a biomarker in the musculoskeletal system. In postmenopausal women, irisin can be used as a

\section{REFERENCES}

Ali, T., Lam, D., Bronze, M. S., and Humphrey, M. B. (2009). Osteoporosis in inflammatory bowel disease. Am. J. Med. 122, 599-604.

Amin, A., Dhillo, W. S., and Murphy, K. G. (2011). The central effects of thyroid hormones on appetite. J. Thyroid. Res. 2011:306510.

Anastasilakis, A. D., Polyzos, S. A., Saridakis, Z. G., Kynigopoulos, G., Skouvaklidou, E. C., Molyvas, D., et al. (2014). Circulating irisin in healthy, young individuals: day-night rhythm, effects of food intake and exercise, and associations with gender, physical activity, diet, and body composition. J. Clin. Endocrinol. Metab. 99, 3247-3255. doi: 10.1210/jc.20141367

Andrade, E. F., Silva, V. O., Moura, N. O., Foureaux, R. C., Orlando, D. R., Moura, R. F., et al. (2018). Physical Exercise Improves Glycemic and Inflammatory Profile and Attenuates Progression of Periodontitis in Diabetic Rats (HFD/STZ). Nutrients 10:1702. doi: 10.3390/nu10111702 biomarker for sarcopenia and hip fracture, because irisin is inversely related to the degree of muscle wasting and the risk of hip fractures (Yan et al., 2018; Park et al., 2019; Ruan et al., 2020). In body composition of children, Irisin is also used as a biomarker owing to the positive correlation with BMD, regardless of lean or fat body mass (Eloranta et al., 2018). Remarkably, according to multiple regression analysis, irisin is even a stronger determinant of bone mineral status than bone alkaline phosphatase (Colaianni et al., 2019).

\section{SUMMARY}

Since the discovery of irisin, owing to many findings relevant to bone metabolism, irisin has attracted much attention. In exercisemediated bone metabolism, irisin ameliorates bone metabolism by regulating muscle and bone cells, modulates the expression of cell factors, and alleviates bone loss under pathological conditions, as shown in Table $\mathbf{1}$ and Figure 1. Interesting, irisin is expected to serve as a biomarker for detecting bone metabolism. Finally, exercise is beneficial for maintaining bone health partly via regulation of irisin, and this may also apply to people with underlying pathological conditions.

\section{AUTHOR CONTRIBUTIONS}

JX and LL conceptualized the review. LL, JG, and XC wrote the manuscript. XT prepared the figure and table. JX and JZ critically reviewed and edited the manuscript. All authors contributed to the article and approved the submitted version.

\section{FUNDING}

This work was supported by funding from National Natural Science Foundation of China (No. 81871835) and Shanghai Key Laboratory of Human Sport Competence Development and Maintenance (Shanghai University of Sport, No. 11DZ2261100), and Australian Health and Medical Research Council (NHMRC, Nos. 1107828 and 1127156).

Archundia-Herrera, C., Macias-Cervantes, M., Ruiz-Munoz, B., Vargas-Ortiz, K., Kornhauser, C., and Perez-Vazquez, V. (2017). Muscle irisin response to aerobic vs HIIT in overweight female adolescents. Diabetol. Metab. Syndr. 9:101.

Beck, B. R., Daly, R. M., Singh, M. A., and Taaffe, D. R. (2017). Exercise and Sports Science Australia (ESSA) position statement on exercise prescription for the prevention and management of osteoporosis. J. Sci. Med. 20, 438-445. doi: 10.1016/j.jsams.2016.10.001

Belviranli, M., and Okudan, N. (2018). Exercise training increases cardiac, hepatic and circulating levels of brain-derived neurotrophic factor and irisin in young and aged rats. Horm. Mol. Biol. Clin. Investig 36:3.

Benedetti, M. G., Furlini, G., Zati, A., and Letizia Mauro, G. (2018). The Effectiveness of Physical Exercise on Bone Density in Osteoporotic Patients. Biomed. Res. Int. 2018:4840531.

Bogl, L. H., Latvala, A., Kaprio, J., Sovijarvi, O., Rissanen, A., and Pietilainen, K. H. (2011). An investigation into the relationship between soft tissue body 
composition and bone mineral density in a young adult twin sample. J. Bone Miner. Res. 26, 79-87. doi: 10.1002/jbmr.192

Bonewald, L. (2019). Use it or lose it to age: a review of bone and muscle communication. Bone 120, 212-218. doi: 10.1016/j.bone.2018.11.002

Bonewald, L. F. (2011). The amazing osteocyte. J. Bone Miner. Res. 26, 229-238. doi: $10.1002 /$ jbmr.320

Bostrom, P., Wu, J., Jedrychowski, M. P., Korde, A., Ye, L., Lo, J. C., et al. (2012). A PGC1- $\alpha$-dependent myokine that drives brown-fat-like development of white fat and thermogenesis. Nature 481, 463-468. doi: 10.1038/nature10777

Camerino, C., Conte, E., Cannone, M., Caloiero, R., Fonzino, A., and Tricarico, D. (2016). Nerve Growth Factor, Brain-Derived Neurotrophic Factor and Osteocalcin Gene Relationship in Energy Regulation, Bone Homeostasis and Reproductive Organs Analyzed by mRNA Quantitative Evaluation and Linear Correlation Analysis. Front. Physiol. 7:456. doi: 10.3389/fphys.2016.00456

Cardozo, C. P., and Graham, Z. A. (2017). Muscle-bone interactions: movement in the field of mechano-humoral coupling of muscle and bone. Ann. N. Y. Acad. Sci. 1402, 10-17. doi: 10.1111/nyas.13411

Chen, X., Sun, K., Zhao, S., Geng, T., Fan, X., Sun, S., et al. (2020). Irisin promotes osteogenic differentiation of bone marrow mesenchymal stem cells by activating autophagy via the Wnt//beta-catenin signal pathway. Cytokine 136:155292. doi: 10.1016/j.cyto.2020.155292

Cheung, A. M., and Giangregorio, L. (2012). Mechanical stimuli and bone health: what is the evidence? Curr. Opin. Rheumatol. 24, 561-566. doi: 10.1097/bor. 0b013e3283570238

Clarke, B. (2008). Normal bone anatomy and physiology. Clin. J. Am. Soc. Nephrol. 3, S131-S139.

Colaianni, G., Cuscito, C., Mongelli, T., Pignataro, P., Buccoliero, C., Liu, P., et al. (2015). The myokine irisin increases cortical bone mass. Proc. Natl. Acad. Sci. U. S. A. 112, 12157-12162. doi: 10.1073/pnas.1516622112

Colaianni, G., Errede, M., Sanesi, L., Notarnicola, A., Celi, M., Zerlotin, R., et al. (2021). Irisin correlates positively with BMD in a cohort of older adult patients and downregulates the senescent marker p21 in osteoblasts. J. Bone Miner. Res. 36, 305-314. doi: 10.1002/jbmr.4192

Colaianni, G., Faienza, M. F., Sanesi, L., Brunetti, G., Pignataro, P., Lippo, L., et al. (2019). Irisin serum levels are positively correlated with bone mineral status in a population of healthy children. Pediatr. Res. 85, 484-488. doi: 10.1038/s41390019-0278-y

Colaianni, G., Mongelli, T., Cuscito, C., Pignataro, P., Lippo, L., Spiro, G., et al. (2017). Irisin prevents and restores bone loss and muscle atrophy in hind-limb suspended mice. Sci. Rep. 7:2811.

Compston, J., Cooper, A., Cooper, C., Gittoes, N., Gregson, C., Harvey, N., et al. (2017). UK clinical guideline for the prevention and treatment of osteoporosis. Arch. Osteoporos 12:43.

Cunningham, H. C., West, D. W. D., Baehr, L. M., Tarke, F. D., Baar, K., Bodine, S. C., et al. (2018). Age-dependent bone loss and recovery during hindlimb unloading and subsequent reloading in rats. BMC Musculoskelet Disord. 19:223. doi: 10.1186/s12891-018-2156-x

Devaraja, J., Jacques, R., Paggiosi, M., Clark, C., and Dimitri, P. (2020). Impact of Type 1 Diabetes Mellitus on Skeletal Integrity and Strength in Adolescents as Assessed by HRpQCT. JBMR Plus 4:e10422.

Dieli-Conwright, C. M., Courneya, K. S., Demark-Wahnefried, W., Sami, N., Lee, K., Sweeney, F. C., et al. (2018). Aerobic and resistance exercise improves physical fitness, bone health, and quality of life in overweight and obese breast cancer survivors: a randomized controlled trial. Breast Cancer Res. 20:124.

Dimitri, P., and Rosen, C. (2017). The Central Nervous System and Bone Metabolism: an Evolving Story. Calcif. Tissue Int. 100, 476-485. doi: 10.1007/ s00223-016-0179-6

Dolan, E., Swinton, P. A., Sale, C., Healy, A., and O'reilly, J. (2017). Influence of adipose tissue mass on bone mass in an overweight or obese population: systematic review and meta-analysis. Nutr. Rev. 75, 858-870. doi: 10.1093/ nutrit/nux046

Ducy, P., Amling, M., Takeda, S., Priemel, M., Schilling, A. F., Beil, F. T., et al. (2000). Leptin inhibits bone formation through a hypothalamic relay: a central control of bone mass. Cell 100, 197-207. doi: 10.1016/s0092-8674(00)81558-5

Elizondo-Montemayor, L., Mendoza-Lara, G., Gutierrez-Delbosque, G., PeschardFranco, M., Nieblas, B., and Garcia-Rivas, G. (2018). Relationship of Circulating Irisin with Body Composition, Physical Activity, and Cardiovascular and Metabolic Disorders in the Pediatric Population. Int. J. Mol. Sci. 19:3727. doi: $10.3390 /$ ijms 19123727
Eloranta, A. M., Jaaskelainen, J., Venalainen, T., Jalkanen, H., Kiiskinen, S., Mantyselka, A., et al. (2018). Birth weight is associated with dietary factors at the age of 6-8 years: the Physical Activity and Nutrition in Children (PANIC) study. Publ. Health Nutr. 21, 1278-1285. doi: 10.1017/s1368980017004013

Faienza, M. F., Brunetti, G., Sanesi, L., Colaianni, G., Celi, M., Piacente, L., et al. (2018). High irisin levels are associated with better glycemic control and bone health in children with Type 1 diabetes. Diabetes Res. Clin. Pract. 141, 10-17. doi: 10.1016/j.diabres.2018.03.046

Farmer, S. R. (2019). Boning Up on Irisin. N. Engl. J. Med. 380, 1480-1482. doi: 10.1056/nejmcibr1900041

Farr, J. N., Chen, Z., Lisse, J. R., Lohman, T. G., and Going, S. B. (2010). Relationship of total body fat mass to weight-bearing bone volumetric density, geometry, and strength in young girls. Bone 46, 977-984. doi: 10.1016/j.bone. 2009.12.033

Farr, J. N., and Dimitri, P. (2017). The Impact of Fat and Obesity on Bone Microarchitecture and Strength in Children. Calcif. Tissue Int. 100, 500-513. doi: 10.1007/s00223-016-0218-3

Fuusager, G. B., Christesen, H. T., Milandt, N., and Schou, A. J. (2019). Glycemic control and bone mineral density in children and adolescents with type 1 diabetes. Pediatr. Diabetes 20, 629-636.

Gamal, R. M., Mohamed, M. E., Hammam, N., El Fetoh, N. A., Rashed, A. M., and Furst, D. E. (2019). Preliminary study of the association of serum irisin levels with poor sleep quality in rheumatoid arthritis patients. Sleep Med. 67, 71-76. doi: 10.1016/j.sleep.2019.10.021

Gomez-Bruton, A., Matute-Llorente, A., Gonzalez-Aguero, A., Casajus, J. A., and Vicente-Rodriguez, G. (2017). Plyometric exercise and bone health in children and adolescents: a systematic review. World J. Pediatr. 13, 112-121. doi: 10. 1007/s12519-016-0076-0

Gouda, W., Mageed, L., Shaker, Y., Hamimy, W. I., and Afify, M. (2018). Assessment of Serum Vitamin D and Irisin Levels in Obese Patients. Clin. Lab 11:64.

Goulding, A., Taylor, R. W., Jones, I. E., Mcauley, K. A., Manning, P. J., and Williams, S. M. (2000). Overweight and obese children have low bone mass and area for their weight. Int. J. Obes. Relat. Metab. Disord. 24, 627-632. doi: 10.1038/sj.ijo.0801207

Handschin, C., and Spiegelman, B. M. (2008). The role of exercise and PGC1alpha in inflammation and chronic disease. Nature 454, 463-469. doi: 10.1038/ nature07206

He, W., Wang, P., Chen, Q., and Li, C. (2020). Exercise enhances mitochondrial fission and mitophagy to improve myopathy following critical limb ischemia in elderly mice via the PGC1a/FNDC5/irisin pathway. Skelet Muscle 10:25.

Houweling, P., Kulkarni, R. N., and Baldock, P. A. (2015). Neuronal control of bone and muscle. Bone 80, 95-100. doi: 10.1016/j.bone.2015.05.006

Huang, L., Yan, S., Luo, L., and Yang, L. (2019). Irisin regulates the expression of BDNF and glycometabolism in diabetic rats. Mol. Med. Rep. 19, 1074-1082.

Idelevich, A., and Baron, R. (2018). Brain to bone: what is the contribution of the brain to skeletal homeostasis? Bone 115, 31-42. doi: 10.1016/j.bone.2018.05.018

Jang, H. B., Kim, H. J., Kang, J. H., Park, S. I., Park, K. H., and Lee, H. J. (2017). Association of circulating irisin levels with metabolic and metabolite profiles of Korean adolescents. Metabolism 73, 100-108. doi: 10.1016/j.metabol.2017.05. 007

Janik, M., Stuss, M., Michalska-Kasiczak, M., Jegier, A., and Sewerynek, E. (2018). Effects of physical activity on sclerostin concentrations. Endokrynol. Pol. 69, 142-149.

Jin, W., Li, Y., Yu, S., Yang, H., and Qian, Z. (1999). [Detecton of circulating antigen in sera from mice infected with Toxoplasma tachyzoites]. Zhongguo Ji Sheng Chong Xue Yu Ji Sheng Chong Bing Za Zhi 17, $117-118$.

Judex, S., and Rubin, C. T. (2010). Is bone formation induced by high-frequency mechanical signals modulated by muscle activity? J. Muscul. Neuronal. Interact. $10,3-11$.

Kang, Y. S., Kim, J. C., Kim, J. S., and Kim, S. H. (2019). Effects of Swimming Exercise on Serum Irisin and Bone FNDC5 in Rat Models of High-Fat Diet-Induced Osteoporosis. J. Sports Sci. Med. 18, 596-603.

Karsenty, G. (2006). Convergence between bone and energy homeostases: leptin regulation of bone mass. Cell Metab. 4, 341-348. doi: 10.1016/j.cmet.2006.10. 008 
Kawao, N., Moritake, A., Tatsumi, K., and Kaji, H. (2018). Roles of Irisin in the Linkage from Muscle to Bone During Mechanical Unloading in Mice. Calcif. Tissue Int. 103, 24-34. doi: 10.1007/s00223-018-0387-3

Kemmler, W., Bebenek, M., Kohl, M., and Von Stengel, S. (2015). Exercise and fractures in postmenopausal women. Final results of the controlled Erlangen Fitness and Osteoporosis Prevention Study (EFOPS). Osteoporos. Int. 26, 24912499. doi: 10.1007/s00198-015-3165-3

Kilian, O., Hartmann, S., Dongowski, N., Karnati, S., Baumgart-Vogt, E., Hartel, F. V., et al. (2014). BDNF and its TrkB receptor in human fracture healing. Ann. Anat. 196, 286-295. doi: 10.1016/j.aanat.2014.06.001

Kim, H. J., Lee, H. J., So, B., Son, J. S., Yoon, D., and Song, W. (2016). Effect of aerobic training and resistance training on circulating irisin level and their association with change of body composition in overweight/obese adults: a pilot study. Physiol. Res. 65, 271-279. doi: 10.33549/physiolres.932997

Kim, J. H., and Kim, D. Y. (2018). Aquarobic exercises improve the serum blood irisin and brain-derived neurotrophic factor levels in elderly women. Exp. Gerontol. 104, 60-65. doi: 10.1016/j.exger.2018.01.024

Kim, H., Wrann, C. D., Jedrychowski, M., Vidoni, S., Kitase, Y., Nagano, K., et al. (2018). Irisin mediates effects on bone and fat via $\alpha \mathrm{v}$ integrin receptors. Cell 175 , 1756-1768. doi: 10.1016/j.cell.2018.10.025

Klein-Nulend, J., Bacabac, R. G., and Bakker, A. D. (2012). Mechanical loading and how it affects bone cells: the role of the osteocyte cytoskeleton in maintaining our skeleton. Eur. Cell Mater 24, 278-291. doi: 10.22203/ecm.v024a20

Klein-Nulend, J., Van Der Plas, A., Semeins, C. M., Ajubi, N. E., Frangos, J. A., Nijweide, P. J., et al. (1995). Sensitivity of osteocytes to biomechanical stress in vitro. FASEB J. 9, 441-445. doi: 10.1096/fasebj.9.5.7896017

Knothe Tate, M. L., Steck, R., Forwood, M. R., and Niederer, P. (2000). In vivo demonstration of load-induced fluid flow in the rat tibia and its potential implications for processes associated with functional adaptation. J. Exp. Biol. 203, 2737-2745.

Kowianski, P., Lietzau, G., Czuba, E., Waskow, M., Steliga, A., and Morys, J. (2018). BDNF: a Key Factor with Multipotent Impact on Brain Signaling and Synaptic Plasticity. Cell Mol. Neurobiol. 38, 579-593. doi: 10.1007/s10571-0170510-4

Kular, J., Tickner, J., Chim, S. M., and Xu, J. (2012). An overview of the regulation of bone remodelling at the cellular level. Clin. Biochem. 45, 863-873. doi: 10.1016/j.clinbiochem.2012.03.021

Kulkarni, R. N., Bakker, A. D., Gruber, E. V., Chae, T. D., Veldkamp, J. B., Klein-Nulend, J., et al. (2012). MT1-MMP modulates the mechanosensitivity of osteocytes. Biochem. Biophys. Res. Commun. 417, 824-829. doi: 10.1016/j. bbrc.2011.12.045

Kurdiova, T., Balaz, M., Mayer, A., Maderova, D., Belan, V., Wolfrum, C., et al. (2014). Exercise-mimicking treatment fails to increase Fndc5 mRNA \& irisin secretion in primary human myotubes. Peptides 56, 1-7. doi: 10.1016/j. peptides.2014.03.003

Lewiecki, E. M. (2011). Sclerostin: a novel target for intervention in the treatment of osteoporosis. Discov. Med. 12, 263-273.

Liu, Q., Lei, L., Yu, T., Jiang, T., and Kang, Y. (2018). Effect of BrainDerived Neurotrophic Factor on the Neurogenesis and Osteogenesis in Bone Engineering. Tissue Eng. Part A 24, 1283-1292. doi: 10.1089/ten.tea.2017. 0462

Liu, T. Y., Shi, C. X., Gao, R., Sun, H. J., Xiong, X. Q., Ding, L., et al. (2015). Irisin inhibits hepatic gluconeogenesis and increases glycogen synthesis via the PI3K/Akt pathway in type 2 diabetic mice and hepatocytes. Clin. Sci. 129, 839-850. doi: 10.1042/cs20150009

Liu, X., Liang, Y., Xia, N., Liu, W., Yang, Q., and Wang, C. (2021). Decrease in leptin mediates rat bone metabolism impairments during high-fat diet-induced catch-up growth by modulating the OPG/RANKL balance. 3 Biotech. 11:103.

Luo, Y., Qiao, X., Ma, Y., Deng, H., Xu, C. C., and Xu, L. (2020). Disordered metabolism in mice lacking irisin. Sci. Rep. 10:17368.

Ma, E. B., Sahar, N. E., Jeong, M., and Huh, J. Y. (2019). Irisin Exerts Inhibitory Effect on Adipogenesis Through Regulation of Wnt Signaling. Front. Physiol. 10:1085. doi: 10.3389/fphys.2019.01085

Mahapatra, C., Singh, R. K., Kim, J. J., Patel, K. D., Perez, R. A., Jang, J. H., et al. (2016). Osteopromoting Reservoir of Stem Cells: bioactive Mesoporous Nanocarrier/Collagen Gel through Slow-Releasing FGF18 and the Activated
BMP Signaling. ACS Appl. Mater Interf. 8, 27573-27584. doi: 10.1021/acsami. 6b09769

Martinez Munoz, I. Y., Camarillo Romero, E. D. S., Correa Padilla, T., Santillan Benitez, J. G., Camarillo Romero, M. D. S., Montenegro Morales, L. P., et al. (2019). Association of Irisin Serum Concentration and Muscle Strength in Normal-Weight and Overweight Young Women. Front. Endocrinol. 10:621. doi: $10.3389 /$ fendo.2019.00621

Mazur-Bialy, A. I., Pochec, E., and Zarawski, M. (2017). Anti-Inflammatory Properties of Irisin, Mediator of Physical Activity, Are Connected with TLR4/MyD88 Signaling Pathway Activation. Int. J. Mol. Sci. 18:701. doi: 10. 3390/ijms18040701

Metzger, C. E., Anand Narayanan, S., Phan, P. H., and Bloomfield, S. A. (2020). Hindlimb unloading causes regional loading-dependent changes in osteocyte inflammatory cytokines that are modulated by exogenous irisin treatment. NPJ Microgr. 6:28.

Metzger, C. E., Narayanan, S. A., Elizondo, J. P., Carter, A. M., Zawieja, D. C., Hogan, H. A., et al. (2019). DSS-induced colitis produces inflammation-induced bone loss while irisin treatment mitigates the inflammatory state in both gut and bone. Sci. Rep. 9:15144.

Morelli, C., Avolio, E., Galluccio, A., Caparello, G., Manes, E., Ferraro, S., et al. (2020). Impact of Vigorous-Intensity Physical Activity on Body Composition Parameters, Lipid Profile Markers, and Irisin Levels in Adolescents: a CrossSectional Study. Nutrients 12:742. doi: 10.3390/nu12030742

Motyl, K. J., and Rosen, C. J. (2012). Understanding leptin-dependent regulation of skeletal homeostasis. Biochimie 94, 2089-2096. doi: 10.1016/j.biochi.2012. 04.015

Murawska-Cialowicz, E., Wojna, J., and Zuwala-Jagiello, J. (2015). Crossfit training changes brain-derived neurotrophic factor and irisin levels at rest, after wingate and progressive tests, and improves aerobic capacity and body composition of young physically active men and women. J. Physiol. Pharmacol. 66, $811-821$.

Nordstrom, P., Pettersson, U., and Lorentzon, R. (1998). Type of physical activity, muscle strength, and pubertal stage as determinants of bone mineral density and bone area in adolescent boys. J. Bone Miner. Res. 13, 1141-1148. doi: 10.1359/jbmr.1998.13.7.1141

Novack, D. V. (2003). TSH, the bone suppressing hormone. Cell 115, 129-130. doi: 10.1016/s0092-8674(03)00812-2

Nygaard, H., Slettalokken, G., Vegge, G., Hollan, I., Whist, J. E., Strand, T., et al. (2015). Irisin in blood increases transiently after single sessions of intense endurance exercise and heavy strength training. PLoS One 10:e0121367. doi: 10.1371/journal.pone.0121367

Okubo, R., Sanada, L. S., Castania, V. A., Louzada, M. J., De Paula, F. J., Maffulli, N., et al. (2017). Jumping exercise preserves bone mineral density and mechanical properties in osteopenic ovariectomized rats even following established osteopenia. Osteoporos Int. 28, 1461-1471. doi: 10.1007/s00198017-3905-7

Palacios-Gonzalez, B., Vadillo-Ortega, F., Polo-Oteyza, E., Sanchez, T., AnciraMoreno, M., Romero-Hidalgo, S., et al. (2015). Irisin levels before and after physical activity among school-age children with different BMI: a direct relation with leptin. Obesity 23, 729-732. doi: 10.1002/oby.21029

Palermo, A., Sanesi, L., Colaianni, G., Tabacco, G., Naciu, A. M., Cesareo, R., et al. (2019). A Novel Interplay Between Irisin and PTH: from Basic Studies to Clinical Evidence in Hyperparathyroidism. J. Clin. Endocrinol. Metab. 104, 3088-3096. doi: 10.1210/jc.201802216

Park, H. S., Kim, H. C., Zhang, D., Yeom, H., and Lim, S. K. (2019). The novel myokine irisin: clinical implications and potential role as a biomarker for sarcopenia in postmenopausal women. Endocrine 64, 341-348. doi: 10.1007/ s12020-018-1814-y

Pedersen, B. K. (2009). The diseasome of physical inactivity-and the role of myokines in muscle-fat cross talk. J. Physiol. 587, 5559-5568. doi: 10.1113/ jphysiol.2009.179515

Pena-Bello, L., Pertega-Diaz, S., Sangiao-Alvarellos, S., Outeirino-Blanco, E., EirasLeal, R., Varela-Rodriguez, B., et al. (2016). Circulating Levels of Irisin in Hypopituitary and Normal Subjects. PLoS One 11:e0160364. doi: 10.1371/ journal.pone.0160364 
Poretsky, L., Islam, J., Avtanski, D., Lin, Y. K., Shen, Y. L., Hirth, Y., et al. (2017). Reproductive effects of irisin: initial in vitro studies. Reprod. Biol. 17, 285-288. doi: 10.1016/j.repbio.2017.05.011

Qiao, X., Nie, Y., Ma, Y., Chen, Y., Cheng, R., Yin, W., et al. (2016). Irisin promotes osteoblast proliferation and differentiation via activating the MAP kinase signaling pathways. Sci. Rep. 6:18732.

Rashid, F. A., Abbas, H. J., Naser, N. A., and Addai Ali, H. (2020). Effect of LongTerm Moderate Physical Exercise on Irisin between Normal Weight and Obese Men. Scient. World J. 2020:1897027.

Rashti, B. A., Mehrabani, J., Damirchi, A., and Babaei, P. (2019). The influence of concurrent training intensity on serum irisin and abdominal fat in postmenopausal women. Prz. Menop. 18, 166-173. doi: 10.5114/pm.2019. 90810

Rattanakul, C., Lenbury, Y., Krishnamara, N., and Wollkind, D. J. (2003). Modeling of bone formation and resorption mediated by parathyroid hormone: response to estrogen/PTH therapy. Biosystems 70, 55-72. doi: 10.1016/s0303-2647(03) 00040-6

Reid, I. R., Baldock, P. A., and Cornish, J. (2018). Effects of Leptin on the Skeleton. Endocr. Rev. 39, 938-959. doi: 10.1210/er.2017-00226

Reza, M. M., Sim, C. M., Subramaniyam, N., Ge, X., Sharma, M., Kambadur, R., et al. (2017a). Irisin treatment improves healing of dystrophic skeletal muscle. Oncotarget 8, 98553-98566. doi: 10.18632/oncotarget.21636

Reza, M. M., Subramaniyam, N., Sim, C. M., Ge, X., Sathiakumar, D., Mcfarlane, C., et al. (2017b). Irisin is a pro-myogenic factor that induces skeletal muscle hypertrophy and rescues denervation-induced atrophy. Nat. Commun. 8:1104.

Robinson, J. A., Chatterjee-Kishore, M., Yaworsky, P. J., Cullen, D. M., Zhao, W., Li, C., et al. (2006). Wnt/beta-catenin signaling is a normal physiological response to mechanical loading in bone. J. Biol. Chem. 281, 31720-31728.

Rodriguez, A., Becerril, S., Mendez-Gimenez, L., Ramirez, B., Sainz, N., Catalan, V., et al. (2015). Leptin administration activates irisin-induced myogenesis via nitric oxide-dependent mechanisms, but reduces its effect on subcutaneous fat browning in mice. Int. J. Obes. 39, 397-407. doi: 10.1038/ijo.2014.166

Ruan, Q., Huang, Y., Yang, L., Ruan, J., Gu, W., Zhang, X., et al. (2019). The effects of both age and sex on irisin levels in paired plasma and cerebrospinal fluid in healthy humans. Peptides 113, 41-51. doi: 10.1016/j.peptides.2019.01.004

Ruan, Q., Huang, Y., Yang, L., Li, J., Gu, W., Bao, Z., et al. (2020). Associations of Preoperative Irisin Levels of Paired Cerebrospinal Fluid and Plasma with Physical Dysfunction and Muscle Wasting Severity in Residents of Surgery Wards. J. Nutr. Health Aging 24, 412-422. doi: 10.1007/s12603-020-1343-2

Sadeghipour, H. R., Yeganeh, G., Zar, A., Salesi, M., Akbarzadeh, S., and Bernardi, M. (2020). The effect of 4-week endurance training on serum levels of irisin and betatrophin in streptozotocin-induced diabetic rats. Arch. Physiol. Biochem. 3, 1-7. doi: 10.1080/13813455.2020.1849310

Scalzo, R. L., Peltonen, G. L., Giordano, G. R., Binns, S. E., Klochak, A. L., Paris, H. L., et al. (2014). Regulators of human white adipose browning: evidence for sympathetic control and sexual dimorphic responses to sprint interval training. PLoS One 9:e90696. doi: 10.1371/journal.pone.0090696

Singh, A., Gupta, M. K., and Mishra, S. P. (2019). Study of correlation of level of expression of Wnt signaling pathway inhibitors sclerostin and dickkopf-1 with disease activity and severity in rheumatoid arthritis patients. Drug. Discov. Ther. 13, 22-27. doi: 10.5582/ddt.2019.01011

Soch, A., Bradburn, S., Sominsky, L., De Luca, S. N., Murgatroyd, C., and Spencer, S. J. (2016). Effects of exercise on adolescent and adult hypothalamic and hippocampal neuroinflammation. Hippocampus 26, 1435-1446. doi: 10.1002/ hipo. 22620

Storlino, G., Colaianni, G., Sanesi, L., Lippo, L., Brunetti, G., Errede, M., et al. (2019). Irisin Prevents Disuse-Induced Osteocyte Apoptosis. J. Bone Miner. Res. 35, 766-775. doi: 10.1002/jbmr.3944

Straub, R. H., Cutolo, M., and Pacifici, R. (2015). Evolutionary medicine and bone loss in chronic inflammatory diseases-A theory of inflammation-related osteopenia. Semin. Arthritis Rheum. 45, 220-228. doi: 10.1016/j.semarthrit. 2015.04.014

Tekin, S., Erden, Y., Ozyalin, F., Cigremis, Y., Colak, C., and Sandal, S. (2017). The effects of intracerebroventricular infusion of irisin on feeding behaviour in rats. Neurosci. Lett. 645, 25-32. doi: 10.1016/j.neulet.2017.02.066
Tenorio, B., Jimenez, T., Barrera, G., Hirsch, S., De La Maza, M. P., Troncoso, R., et al. (2017). Irisin is weakly associated with usual physical activity in young overweight women. Nutr. Hosp. 34, 688-692. doi: 10.20960/nh.463

Torre-Saldana, V. A., Gomez-Samano, M. A., Gomez-Perez, F. J., Rosas-Saucedo, J., Leon-Suarez, A., Grajales-Gomez, M., et al. (2019). Fasting Insulin and Alanine Amino Transferase, but Not Fgf21, Were Independent Parameters Related with Irisin Increment after Intensive Aerobic Exercising. Rev. Invest. Clin. 71, 133-140.

Troy, K. L., Mancuso, M. E., Butler, T. A., and Johnson, J. E. (2018). Exercise Early and Often: Effects of Physical Activity and Exercise on Women's Bone Health. Int. J. Environ. Res. Publ. Health 15:878. doi: 10.3390/ijerph15050878

Tsuchiya, Y., Ando, D., Goto, K., Kiuchi, M., Yamakita, M., and Koyama, K. (2014). High-intensity exercise causes greater irisin response compared with low-intensity exercise under similar energy consumption. Tohoku J. Exp. Med. 233, 135-140. doi: 10.1620/tjem.233.135

Tsuchiya, Y., Ando, D., Takamatsu, K., and Goto, K. (2015). Resistance exercise induces a greater irisin response than endurance exercise. Metabolism 64, 1042-1050. doi: 10.1016/j.metabol.2015.05.010

Tu, X., Rhee, Y., Condon, K. W., Bivi, N., Allen, M. R., Dwyer, D., et al. (2012). Sost downregulation and local Wnt signaling are required for the osteogenic response to mechanical loading. Bone 50, 209-217. doi: 10.1016/j.bone.2011. 10.025

Usui, T., Maki, K., Toki, Y., Shibasaki, Y., Takanobu, H., Takanishi, A., et al. (2003). Measurement of mechanical strain on mandibular surface with mastication robot: influence of muscle loading direction and magnitude. Orthod. Craniofac. Res. 6, 163-167. doi: 10.1034/j.1600-0544.2003.250.x

van Bezooijen, R. L., Roelen, B. A., Visser, A., Van Der Wee-Pals, L., De Wilt, E., Karperien, M., et al. (2004). Sclerostin is an osteocyte-expressed negative regulator of bone formation, but not a classical BMP antagonist. J. Exp. Med. 199, 805-814. doi: 10.1084/jem.20031454

Watson, S., Weeks, B., Weis, L., Harding, A., Horan, S., and Beck, B. (2019). HighIntensity Resistance and Impact Training Improves Bone Mineral Density and Physical Function in Postmenopausal Women With Osteopenia and Osteoporosis: The LIFTMOR Randomized Controlled Trial. J. Bone Miner. Res. 34:572. doi: 10.1002/jbmr.3659

Weber, D. R., Haynes, K., Leonard, M. B., Willi, S. M., and Denburg, M. R. (2015). Type 1 diabetes is associated with an increased risk of fracture across the life span: a population-based cohort study using The Health Improvement Network (THIN). Diabet. Care 38, 1913-1920. doi: 10.2337/dc15-0783

Wiecek, M., Szymura, J., Maciejczyk, M., Kantorowicz, M., and Szygula, Z. (2018). Acute Anaerobic Exercise Affects the Secretion of Asprosin, Irisin, and Other Cytokines - A Comparison Between Sexes. Front. Physiol. 9:1782. doi: 10.3389/ fphys.2018.01782

Xu, L., Shen, L., Yu, X., Li, P., Wang, Q., and Li, C. (2020). Effects of irisin on osteoblast apoptosis and osteoporosis in postmenopausal osteoporosis rats through upregulating Nrf2 and inhibiting NLRP3 inflammasome. Exp. Ther. Med. 19, 1084-1090.

Yan, J., Liu, H. J., Guo, W. C., and Yang, J. (2018). Low serum concentrations of Irisin are associated with increased risk of hip fracture in Chinese older women. Joint Bone Spine 85, 353-358. doi: 10.1016/j.jbspin.2017. 03.011

Yang, J., Sun, L., Fan, X., Yin, B., Kang, Y., Tang, L., et al. (2018). Effect of exercise on bone in poorly controlled type 1 diabetes mediated by the ActRIIB/Smad signaling pathway. Exp. Ther. Med. 16, 3686-3693.

Yang, L., Zhi, S., Yang, G., Qin, C., Zhao, W., Niu, M., et al. (2020). Molecular identification of FNDC5 and effect of irisin on the glucose metabolism in common carp (Cyprinus carpio L.). Gen. Comp. Endocrinol. 301:113647. doi: 10.1016/j.ygcen.2020.113647

Ye, W., Wang, J., Lin, D., and Ding, Z. (2020). The immunomodulatory role of irisin on osteogenesis via AMPK-mediated macrophage polarization. Int. J. Biol. Macromol. 146, 25-35. doi: 10.1016/j.ijbiomac.2019.12.028

Zengin, A., Pye, S. R., Cook, M. J., Adams, J. E., Rawer, R., Wu, F. C. W., et al. (2017). Associations of muscle force, power, cross-sectional muscle area and bone geometry in older UK men. J. Cachexia Sarcopenia Muscle 8, 598-606. doi: $10.1002 /$ jcsm. 12198 
Zhang, D., Bae, C., Lee, J., Lee, J., Jin, Z., Kang, M., et al. (2018). The bone anabolic effects of irisin are through preferential stimulation of aerobic glycolysis. Bone 114, 150-160. doi: 10.1016/j.bone.2018.05.013

Zhang, J., Huang, X., Yu, R., Wang, Y., and Gao, C. (2020). Circulating irisin is linked to bone mineral density in geriatric Chinese men. Open Med. 15, 763-768. doi: 10.1515/med-2020-0215

Zhang, J., Valverde, P., Zhu, X., Murray, D., Wu, Y., Yu, L., et al. (2017). Exercise-induced irisin in bone and systemic irisin administration reveal new regulatory mechanisms of bone metabolism. Bone Res. 5: 16056.
Conflict of Interest: The authors declare that the research was conducted in the absence of any commercial or financial relationships that could be construed as a potential conflict of interest.

Copyright (c) $2021 \mathrm{Liu}, \mathrm{Guo}$, Chen, Tong, Xu and Zou. This is an open-access article distributed under the terms of the Creative Commons Attribution License (CC BY). The use, distribution or reproduction in other forums is permitted, provided the original author(s) and the copyright owner(s) are credited and that the original publication in this journal is cited, in accordance with accepted academic practice. No use, distribution or reproduction is permitted which does not comply with these terms. 